\title{
A Short-Turn Dispatching Strategy to Improve the Reliability of Bus Operation
}

\author{
Runkun Liu $\mathbb{D D}^{1}{ }^{1}$ Haiyang Yu $\mathbb{D}^{1,2}$ Pengfei Wang $\left(\mathbb{D},{ }^{3,4} \text { and Hai Yan }{ }^{5}\right)^{5}$ \\ ${ }^{1}$ School of Transportation Science and Engineering, \\ Beijing Key Laboratory for Cooperative Vehicle Infrastructure Systems and Safety Control, Beihang University, \\ Beijing 100191, China \\ ${ }^{2}$ Beijing Advanced Innovation Center for Big Data and Brain Computing, Beihang University, Beijing 100191, China \\ ${ }^{3}$ Key Laboratory of Urban Security and Disaster Engineering of Ministry of Education, Beijing University of Technology, \\ Beijing 100124, China \\ ${ }^{4}$ College of Urban Construction, Hebei Normal University of Science \& Technology, Qinhuangdao, Hebei 066004, China \\ ${ }^{5}$ Beijing Key Laboratory of Traffic Engineering, Beijing University of Technology, Beijing 100124, China \\ Correspondence should be addressed to Hai Yan; yhai@bjut.edu.cn
}

Received 16 October 2019; Revised 26 November 2020; Accepted 4 December 2020; Published 15 December 2020

Academic Editor: Tao Liu

Copyright (c) 2020 Runkun Liu et al. This is an open access article distributed under the Creative Commons Attribution License, which permits unrestricted use, distribution, and reproduction in any medium, provided the original work is properly cited.

Bus dwelling time at stops is affected by the number of boarding and alighting passengers. Due to the uncertainty of traffic state, dwelling time will become unstable, resulting in unreliable bus operations. A short-turn strategy shares passenger demand in certain segments of the full-length vehicles; therefore, this strategy can reduce the dwell time of the full-length vehicles and have potential to improve reliability of bus operations. Firstly, this paper builds a bus operation state model considering short-turn vehicles. Secondly, the process of bus operation is analyzed to investigate the impact of passenger demand on reliability. Then, we propose a short-turn dispatching strategy, which can accurately determine the operation area and departure time. Finally, a numerical simulation is used to evaluate different strategies. As the result, the analysis reveals the following: (1) the bus operational reliability of full-length vehicles under high passenger demand could be improved by a well-designed short-turn strategy; (2) it is beneficial to improve the reliability of bus operation when short-turn vehicles departure at the change point; and (3) an undesigned short-turn dispatching strategy has a negative effect on full-length vehicles operational reliability. This study can help design a short-turn strategy and make valuable contributions to the bus management system.

\section{Introduction}

Bus priority development policy is an important transportation strategy to solve traffic problems in large cities and achieves sustainable urban development. Moreover, the reliability of operation greatly affects the level of service on public transport and affects passengers' choices in public transport. Different researchers have different understandings of reliability [1]. Bates et al. [2] associated reliability with public transportation according to timetable operation. Turnquist and Bowman [3] believed that the maintenance of regular service is an important indicator of reliable public transport. In a large city like Beijing, there is no fixed timetable for bus departure. The departure time is based on the real-time traffic state, passenger demand, as well as other factors. For instance, in the morning and evening peak periods, the bus company increases the frequency of the departure. Therefore, reliability in Beijing refers to the stability of bus headway, which can be expressed as the mean absolute error between bus headway and the arrival time interval.

Multiple factors affect the bus operation reliability, such as traffic congestion, weather conditions, time of passengers' boarding and departure, and drivers' operations, which may result in late bus arrival or irregular arrival [4]. In the previous studies, Sorratini et al. [1] and Mark et al. [5] found that the number of passengers and the time they spend on boarding and alighting a bus were the main reasons for unreliability. 
Especially when buses and cars are mixed and traffic flow is saturated, the speed of the buses drops sharply and buses cannot be operated in accordance with established timetables. Large interval and bus bunching will happen. These phenomena make the main contribution to unreliability.

Several methods have been proposed to ease the instability and excessive demand of passengers at some stations. Tétreault and El-Geneidy [6] adopted the express bus service, and Furth [7] adopted short-turn service, and also the bus holding and stop-skipping methods were used $[8,9]$. Express bus service is to skip some stations with lower passenger demand. Once the departure frequency cannot be guaranteed, this method will waste some passengers' time for short trips and reduce the attractiveness of public transport. In terms of the bus holding method, this method also wastes the time of passengers to some extent. Liang et al. [10] proposed a coordinated control method combining two bus holding and stop-skipping methods. By contrast, short-turn services can meet the requirements of the passengers on the higher demand section of the road and effectively alleviate the situations that more passengers waiting on the bus line at some stations. Therefore, short-turn services can greatly affect buses dwell time and then affect the operation state of full-length vehicles.

Prior work has documented short-turn services. But, most of the existing research studies focused on the problem of combined dispatching with multiple services such as short-turn service, full-length service, and express bus service. Most studies focus on building an optimization model to reduce the waiting time of passengers or bus operation costs [11-14]. For example, Tirachini et al. [14] established a scheduling model considering passenger travel cost and the operation cost, and the genetic algorithm was used to solve departure frequency. The operation area of short-turn vehicles and the first and last stops are determined through OD (origin-destination) data with consideration of passenger demand and vehicle capacity in Furth's studies [7, 15]. Canca et al. [16] developed an optimization model which integrates short-turning shuttle operations. Because short-turn vehicles only travel between some certain stations of the line with a limited number of passengers, it is possible to consider using different models to reduce operation cost. For example, Ceder et al. [17] took different sized vehicles to optimize the bus dispatching problem. Within our knowledge, less work has been done to analyze the impact of the short-turn strategy on the public transport system from the perspective of reliability.

In this paper, we focus on designing a simple and effective short-turn strategy to improve bus operational reliability. There are two key factors in designing the shortturn strategy: where to operate and when to depart. For the first factor, it is generally believed that the reasonable operating area of short-turn vehicles should be in the stations with larger passenger demand [18]. Turn-back points should be located with the objective of diminishing the passengers' waiting time while preserving a certain level of service quality. The operation area of short-turn vehicles should satisfy the needs of passengers as much as possible. For the second factor, although the studies listed above showed that most of the scheduling methods of the shortturn vehicles are optimized under the targets of the departure frequency and the minimum passenger travel cost $[19,20]$, it is impossible to accurately determine the reasonable departure time of the short-turn vehicles. We believe the departure time should be in rush hours of the day to release the pressure of full-length vehicles. That means, the departure time of the short-turn vehicles is set based on the surge in passenger demand or the time at which the road reaches congestion (rush hour). That timing is named as change point. Taylor [21] proposed a simple and effective method to analyze the change point, which can accurately locate the change point of a sequence. The study of change point is widely used in economics, meteorology, and other fields. For example, studying economic changes helps discover potential financial risks [22]; observing climate change leads to corresponding countermeasures [23] and so on. Variables in the transportation system such as the speed and the number of passengers are similar to the sequences of economy and climate. The same change point test method can be used to detect the time for traffic congestion and passenger demand surge.

The main contributions of this paper are summarized as the following three points. (1) A simulation-based model of the bus operation state is established, which can describe the real operation process of buses. The causes of bus unreliability are analyzed in detail. (2) A short-turn dispatching strategy is proposed. Through this strategy, the departure time and the operating area of the short-turn vehicles can be set. (3) Different strategies are analyzed by numerical simulation based on the bus operation state model considering short-turn vehicles. The data come from the smart card data of a bus line in Beijing. Results believe that a welldesigned short-turn strategy could improve the reliability of the bus operation.

The reminder of this paper is summarized as follows: in Section 2, the operation process of buses is modeled; in Section 3, we explored the impact of passenger number on the operation state of buses; then, in Section 4, the method of establishing the operating area and departure time of shortturn vehicles is discussed; in Section 5, a simulation experiment is executed with different strategies and the results are analyzed. Finally, Section 6 includes a discussion of the results and concluding thoughts.

\section{Modeling of Bus Operation State}

2.1. Notations. According to the operation process of buses, the operational time of buses consists of two parts: travel time and dwell time (see Figure 1).

We build a mathematical model to describe operation process of full-length buses and short-turn buses, including the bus dwell time, bus arrival time, and departure time in each station. The notations are summarized in Table 1.

This study is based on the following hypotheses:

(i) The assumption of bus dwell time.

The dwell time of buses at each station is assumed only to be related to the number of passengers 


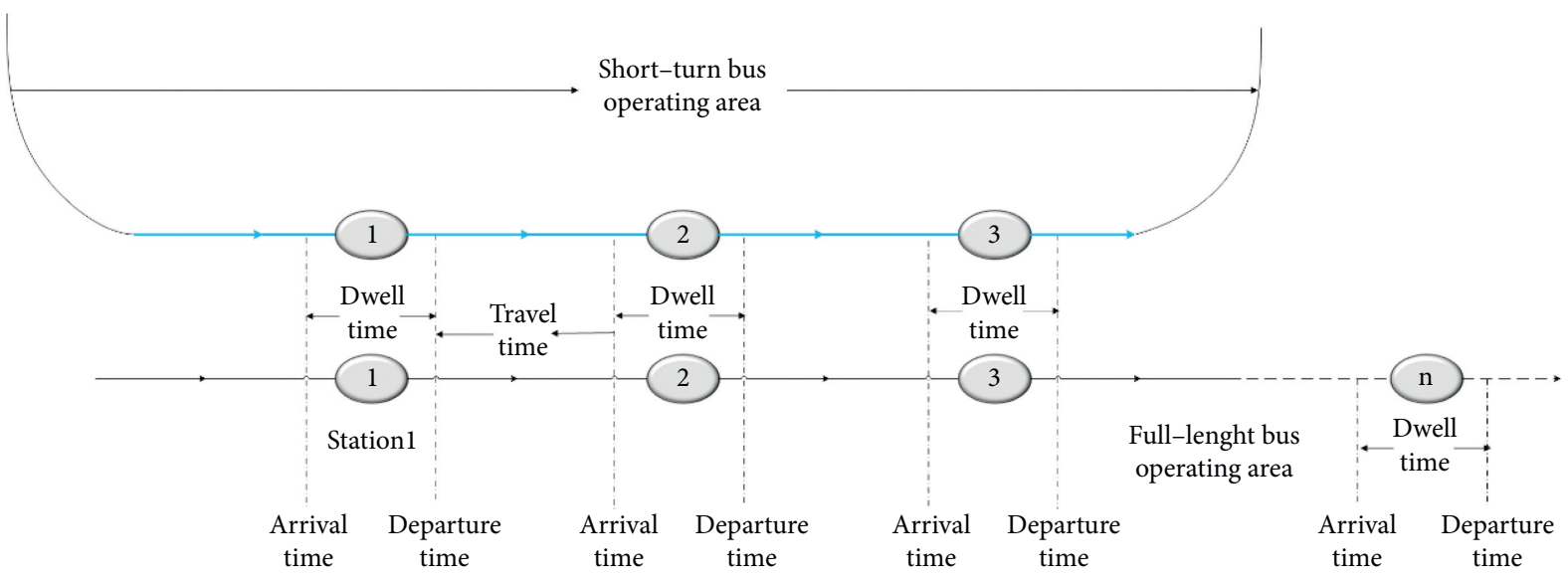

FIGURE 1: Sectional sketch map of bus travel time.

TABLe 1: Notations.

\begin{tabular}{lr}
\hline Notations & Definitions \\
\hline$N$ & Bus index, $\mathrm{n} \in \forall N+, \mathrm{n} \neq 1$ \\
$I$ & Station number, $i \in(1,2,3, \ldots, \mathrm{s}) i \in(1,2,3, \ldots, s)$ \\
$J$ & Interstation index, $j \in(1,2,3, \ldots, s-1) j \in 1,2,3, \ldots, s-1$ \\
$\rho_{i}^{m}$ & Passenger arrival rate at station $i$ in time period $m$ \\
$M$ & Number of periods between to buses \\
$t^{\prime}$ & The time it takes for each passenger to get on/off the bus \\
$\mathrm{TS}_{i}^{n}$ & Dwell time of bus $n$ at station $i$ \\
$Q_{i}^{n}$ & Boarding number of bus $n$ at the station $i$ \\
$V_{j}^{n}$ & The average speed of bus $n$ travel at station interval $j$ \\
$L_{j}$ & The distance of the station intervals $j$ \\
$\mathrm{TT}_{j}^{n}$ & Travel time of bus $n$ at station intervals $j$ \\
$\mathrm{TL}_{k}^{n}$ & The departure time of bus $n$ at station $k$ \\
$\varepsilon$ & A constant for the other time loss required at each station \\
$H_{i}^{n-1, n}$ & Headway of bus $n$ and bus $n$ - 1 at station $i$ (the difference between the departure time of the two buses at the station $i)$ \\
$U$ & Station set of short-turn vehicles operating $U \subseteq(1,2,3, \ldots, s)$ \\
$Q$ & Short-turn bus between full-length buses $n-1$ and $n$ \\
$\mathrm{OD}_{a b}$ & The number of passengers who board at station $a$ and get off at station $b, a, b \in(1,2,3, \ldots, s)$
\end{tabular}

getting on/off, and the maximum time for passengers to get on/off is adopted. The other time loss required for the bus to stop at each station is set as a constant.

(ii) The assumption that all passengers waiting for buses at a station do not need to wait for the bus a second time.

When the short-turn strategy is not applied, all passengers waiting at station $i$ can board the bus [24].

Next, the main parameters of the full-length vehicles' operation state are modeled.

\subsection{The Main Parameters of Full-Length Vehicles}

2.2.1. Bus Dwell Time. When a bus departs from station $i$, if the arrival rate of passengers does not change during period $m$ and they gather at the station $i$ with a rate of $\rho_{i}^{m}$, the number of passengers gathered in station $i$ is $\rho_{i}^{m} \cdot H_{i}^{n-1, n}$ before the next bus arrives. But actually, the arrival rate of passengers changes in real time. If the arrival rate has changed several times within $H_{i}^{n-1, n}$, for example, the arrival rate changes 3 times within $500 \mathrm{~s}$, during $0-200 \mathrm{~s}$ is 2 pax/ $\mathrm{min}, 200-300 \mathrm{~s}$ is $1 \mathrm{pax} / \mathrm{min}$, and $300-500 \mathrm{~s}$ is $2 \mathrm{pax} / \mathrm{min}$. Then, the total number of passengers arriving should be $(200 * 2+100 * 1+200 * 2) / 60=15$. The dwell time is related to the maximum number of passengers getting on and off at station $i$, which can be expressed as follows:

$$
\mathrm{TS}_{i}^{n}=\max \left\{\sum_{a=1}^{i} \mathrm{OD}_{a i}, \sum_{m=1}^{M} \rho_{i}^{m} \cdot H_{i}^{n-1, n, m}\right\} \cdot t^{\prime}+\varepsilon
$$

where $M$ represents the number of arrival rate changes during $H_{i}^{n-1, n}$ and $\rho_{i}^{1} \cdot H_{i}^{n-1, n, 1}$ indicates the cumulative number of passengers before the first arrival rate changed in $H_{i}^{n-1, n}$.

2.2.2. Travel Time between Two Stations. The travel time of buses between adjacent stations is expressed as the distance been divided by speed: 


$$
\mathrm{TT}_{j}^{n}=\frac{L_{j}}{V_{j}^{n}}
$$

2.2.3. Arrival Time. We assume buses arrival at the first station in a fix time interval $T$ and $\mathrm{TA}_{1}^{1}=0$. The arrival time of the bus $n$ at station $k$ is the dwell time plus all the travel time before station $k$ :

$$
\mathrm{TA}_{k}^{n}= \begin{cases}(n-1) T, & \text { if } k=1, \\ \mathrm{TA}_{1}^{n}+\sum_{i=1}^{k-1} \mathrm{TS}_{i}^{n}+\sum_{j=1}^{k-1} \mathrm{TT}_{j}^{n}, & \text { if } 2 \leq k \leq s .\end{cases}
$$

2.2.4. Departure Time. The departure time of the bus can be expressed as the summation of dwell time and travel time of all stations in the previous operation of the bus:

$$
\mathrm{TL}_{k}^{n}= \begin{cases}\mathrm{TA}_{k}^{n}+\mathrm{TS}_{k}^{n}, & \text { if } k=1, \\ \mathrm{TL}_{1}^{n}+\sum_{i=1}^{k} \mathrm{TS}_{i}^{n}+\sum_{j=1}^{k-1} \mathrm{TT}_{j}^{n}, & \text { if } 2 \leq k \leq s .\end{cases}
$$

2.3. The Main Parameters of Short-Turn Vehicles. Based on the notations and two hypotheses in Section 2.1, a state model of short-turn vehicles operation is established. In this study, it is assumed that passengers waiting at the station will give priority to the first arriving vehicle. We define the operating area of a short-turn vehicle as $U$ where $U=\left(u_{1}, u_{2}, K, u_{q}\right), U \subseteq(1,2,3, \ldots, s)$. When the short-turn vehicle arrives at one of the stations, all the passengers whose travel destinations are only in $U$ while the passengers whose destination is not in $U$ will wait for the next full-length vehicle. That means, the passenger whose travel destination is in $U$ will not continue to wait.

2.3.1. Number of Passengers Boarding on Short-Turn Vehicle. We can calculate the probability that the passengers waiting at station $i$ will have a destination within the $U$ range from OD data. The probability model is expressed as follows:

$$
P_{i}=\frac{\sum_{j=i+1}^{u_{q}} \mathrm{OD}_{i j}}{\sum_{j=i+1}^{s} \mathrm{OD}_{i j}}, \quad i \in U .
$$

Then, how many passengers in station $i$ ride the shortturn vehicles can be calculated, and the formula is expressed as follows:

$$
Q_{i}^{q}=P_{i} \cdot \sum_{m=1}^{M} \rho_{i}^{m} \cdot H_{i}^{n-1, q, m}
$$

2.3.2. The Number of Passengers on the Full-Length Vehicle next to a Short-Turn Vehicle. In the study, only alternate departure between the short-turn and full-length vehicle is discussed. The scenario of the continuous short-turn vehicle departure will not be considered. $n q$ stands for the number of short-turn vehicles that departs after $n-1$. Because not all passengers will take the short-turn vehicle, after nq departs from station $i$, some passengers will keep waiting, until bus $n$ arrival. The number of passengers who continue to wait for $n$ is calculated using the following equation:

$$
Q^{q^{\prime}}=\left(1-P_{i}\right) \cdot \sum_{m=1}^{M} \rho_{i}^{m} H_{i}^{n-1, q, m}+\sum_{m^{\prime}=1}^{M^{\prime}} \rho_{i}^{m} H_{i}^{q, n, m^{\prime}},
$$

where $M^{\prime}$ represents the number of arrival rate changes during $H_{i}^{q, n}$.

2.3.3. Dwell Time of Short-Turn Vehicles. The dwell time of a short-turn vehicle at the certain station is expressed as follows:

$$
\mathrm{TS}_{i}^{q}=\max \left\{\sum_{a=u_{1}}^{i} \mathrm{OD}_{a i}, Q_{i}^{q}\right\} \cdot t^{\prime}+\varepsilon, \quad i \in U .
$$

2.3.4. Dwell Time of Full-Length Vehicle next to Short-Turn Vehicles. After a short-turn strategy is applied, the next fulllength vehicle will take fewer passengers. As a result, the dwell time of the full-length vehicles in $U$ will change. When full-length vehicles travel beyond $U$, its dwell time can be calculated by (1). We combine (1) and (8) together, and the dwell time can be expressed as follows:

$$
\mathrm{TS}_{i}^{n}= \begin{cases}\max \left\{\sum_{a=1}^{i} \mathrm{OD}_{a i}, Q_{i}^{q^{\prime}}\right\} \cdot t^{\prime}+\varepsilon, & \text { if } i \in U, \\ \max \left\{\sum_{a=1}^{i} \mathrm{OD}_{a i}, \sum_{m=1}^{M} \rho_{\mathrm{i}}^{m} \cdot H_{\mathrm{i}}^{n-1, n, m}\right\} \cdot t^{\prime}+\varepsilon, & \text { if } i \notin U .\end{cases}
$$

It can be found once the parameters of speed, OD data, and the passenger arrival rate are given in the model. The operational state of full-length vehicles and short-turn vehicles can be calculated. Obviously, since the IC card data can provide information about the three parameters above, it is most rational to use IC card data. In Section 3, we introduced how to extract the data required by the model and analyzed the impact of different factors on the public transportation system.

\section{Bus Operation Analysis without Short-Turn Dispatching Strategy}

3.1. Data Processing. The smart card data of a bus line (No. 973) in Beijing are used to study the impact of the short-turn strategy on the stability of the headway. There are 50 stations on this line. The data are collected from five working days between April 11, 2017 and April 15, 2017, and the weather is sunny. The bus route map is shown in Figure 2.

Original data mainly contain the following fields, and detailed explanation is given in Table 2. 


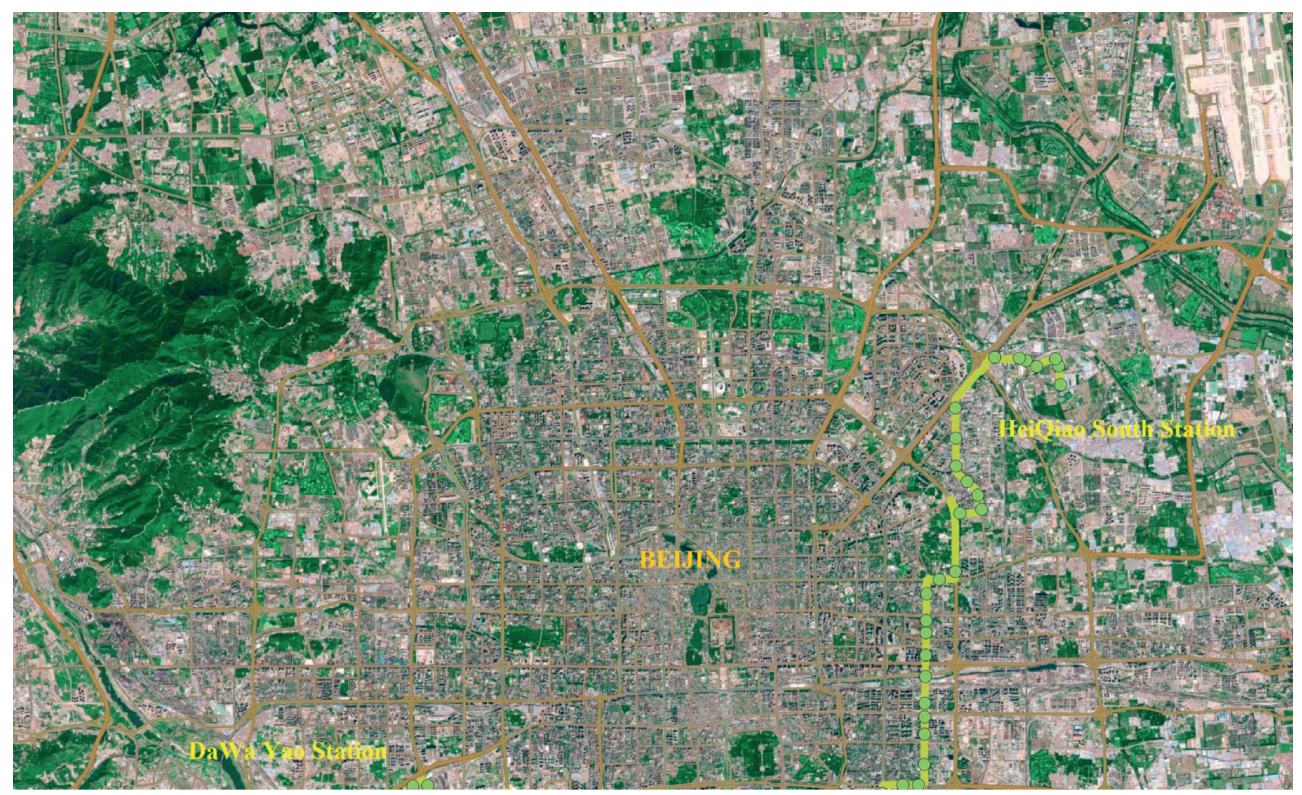

Figure 2: Bus line (sourcing from Amap: https://ditu.amap.com/).

TAвLe 2: Data field description.

\begin{tabular}{lcc}
\hline Field & Type & Comment \\
\hline GRANT_CARD_CODE & String & Card ID \\
LINE_CODE & String & Line code \\
VEHICLE_CODE & String & Vehicle code \\
BANCIID & Int & Shift code \\
ON_STATIONID & Int & Get-on station ID \\
ON_STATIONNAME & String & Get-on station name \\
OFF_STATIONID & String & Get-off station ID \\
OFF_STATIONNAME & String & Get-off station name \\
OFF_STATION_TIME & String & Get-off card time \\
LOC_TREND & Int & Bus operation direction \\
\hline
\end{tabular}

We can find that there is no record of the time in the table when a passenger boarded. Since December 28, 2014, a passenger in Beijing has been charged by mileage in public transport. Passengers need to swipe their cards when getting on and off the bus. In order to save space for information storage, boarding time has not been recorded, and it is useless for charging [25].

The data required for the simulation are extracted.

3.1.1. The Distance of Station Interval. The distance can be calculated with the latitude and longitude of each station, and the result is calibrated by an investigation, and data are shown in Table 3.

3.1.2. Speed Data. The short-turn strategy is usually applied during rush hours. Therefore, we extracted the data of speed and passenger arrival rate from 7 to $10 \mathrm{am}$, including the morning peak.

Firstly, the arrival time of a bus at a station can be regarded as the average time of all card-wiping records when getting off the bus [25]. Based on the arrival time, travel time between the two stations can be calculated by distance and travel time. We divide the daily data by the unit of 10 minutes. Then, we calculate travel speed separately and take the average travel speed of the same group each day. In this way, we can get the average speed of each station interval every 10 minutes. As shown in Table 4, the unit is $\mathrm{m} / \mathrm{s}$ and speed_1 indicates the speed of a bus traveling between each station within the first 10 mins. The data of 4 stations and 5 periods are only listed.

3.1.3. Passenger Arrival Rate Data. The smart card data record the information of the boarding and disembarking sites for each passenger, and the passenger OD data of bus line can be counted. The result is shown in Figure 3.

According to the statistical yearbook published by the Beijing Transportation Operations Coordination Center (TOCC), $85 \%$ of passengers choose to pay by card and $15 \%$ of passengers choose to pay cash. Therefore, the OD data obtained from the card data cannot present a complete map of the number of passengers. We multiplied the OD data by a coefficient of 1.17 to get the final passenger OD data.

Since only get-off time is recorded, passenger's boarding time needs to be supplemented to calculate the arrival rate in each station. Bus arrival time can be regarded as passenger's boarding time, which has been obtained when calculating the speed data. Then, the data are grouped every 10 minutes by boarding time, and the number of passengers in each group can be counted. The number of passengers transferred to the arrival rate data is shown in Table 5, the unit is pax/ min, and time_1 indicates the passenger arrival rate at each station in the first 10 minutes. The data of 4 stations and 5 periods are only listed.

3.2. Numerical Simulation. Three scenarios are designed to discuss the impact of different parameters (bus travel speed and passenger arrival rate) on reliability. In each scenario, 15 
TAble 3: Distance of station interval.

\begin{tabular}{|c|c|}
\hline Interstation index & Distance $(\mathrm{m})$ \\
\hline 1 & 118 \\
\hline 2 & 348 \\
\hline 3 & 827 \\
\hline 4 & 846 \\
\hline 5 & 509 \\
\hline 6 & 539 \\
\hline 7 & 356 \\
\hline 8 & 827 \\
\hline 9 & 270 \\
\hline 10 & 1010 \\
\hline 11 & 432 \\
\hline 12 & 384 \\
\hline 13 & 3075 \\
\hline 14 & 1055 \\
\hline 15 & 1887 \\
\hline 16 & 2004 \\
\hline 17 & 865 \\
\hline 18 & 996 \\
\hline 19 & 1083 \\
\hline 20 & 1506 \\
\hline 21 & 826 \\
\hline 22 & 1669 \\
\hline 23 & 817 \\
\hline 24 & 522 \\
\hline 25 & 792 \\
\hline 26 & 808 \\
\hline 27 & 659 \\
\hline 28 & 643 \\
\hline 29 & 1002 \\
\hline 30 & 353 \\
\hline 31 & 880 \\
\hline 32 & 477 \\
\hline 33 & 868 \\
\hline 34 & 868 \\
\hline 35 & 342 \\
\hline 36 & 2088 \\
\hline 37 & 680 \\
\hline 38 & 462 \\
\hline 39 & 755 \\
\hline 40 & 495 \\
\hline 41 & 886 \\
\hline 42 & 1177 \\
\hline 43 & 2109 \\
\hline 44 & 787 \\
\hline 45 & 155 \\
\hline 46 & 524 \\
\hline 47 & 523 \\
\hline 48 & 338 \\
\hline 49 & 688 \\
\hline
\end{tabular}

TABle 4: Speed data.

\begin{tabular}{lccccc}
\hline $\begin{array}{l}\text { Station interval } \\
\text { ID }\end{array}$ & speed_1 & speed_2 & speed_3 & speed_4 & speed_5 \\
\hline 1 & 5.52 & 5.52 & 5.52 & 5.52 & 5.52 \\
2 & 6.90 & 6.90 & 6.90 & 6.90 & 6.90 \\
3 & 6.90 & 6.90 & 6.90 & 6.90 & 6.90 \\
4 & 5.99 & 5.99 & 5.99 & 5.99 & 5.99 \\
\hline
\end{tabular}

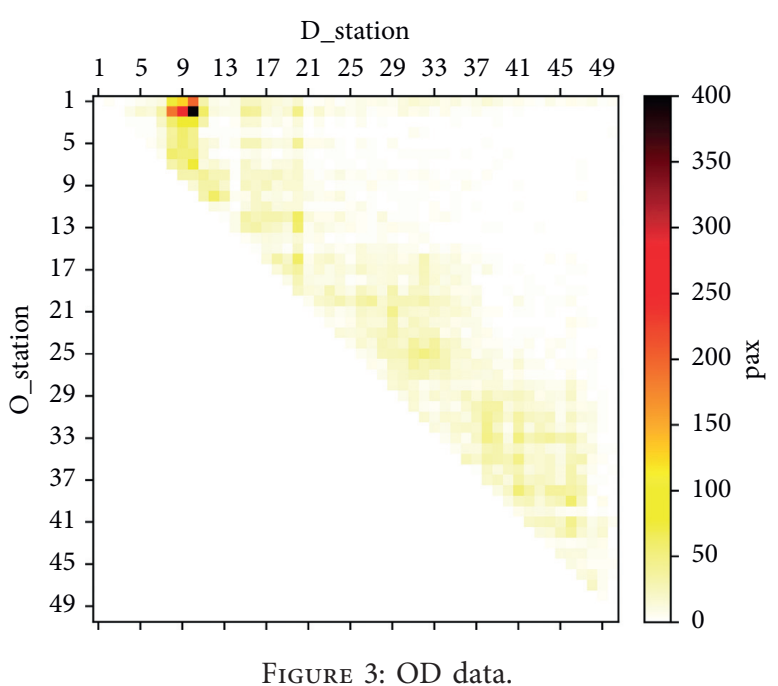

Table 5: Passenger arrival rate.

\begin{tabular}{lccccc}
\hline Station & time_1 & time_2 & time_3 & time_4 & time_5 \\
\hline 1 & 2.98 & 3.22 & 2.04 & 3.47 & 2.98 \\
2 & 3.92 & 4.45 & 3.43 & 4.98 & 3.92 \\
3 & 1.60 & 1.56 & 1.10 & 1.52 & 1.60 \\
4 & 0.76 & 0.70 & 0.79 & 0.64 & 0.76 \\
\hline
\end{tabular}

buses are simulated, and the interval of arrival time is $300 \mathrm{~s}$. The initial time of the system is $0 \mathrm{~s}$. These scenarios are described in Table 6.

The scenarios design is summarized as follows.

Scenario 1 indicates that no control strategy is adopted. It can be considered as a basic scenario.

In scenario 2, bus speed is set to a constant. We are able to assess the impact of passengers' arrival rate changes on bus operation by comparison with scenario 1 .

In scenario 3, the passengers' arrival rate is unchanged with time. We can analyze the impact of speed changes on bus operation by comparison with scenario 1 .

Applying the passenger arrival rate and speed data in the above different scenarios into the model proposed in Section 2.2, we can calculate bus trajectories and bus headway, as shown in Figure 4.

In scenario 1 , we can find the phenomenon of a large interval and bus bunching at some stations from Figure 4(a). This phenomenon indicates that the unstable distribution of bus travel speed and passenger arrival rate makes the contribution with unreliability. Meanwhile, the unreliability increases with travel distance. This phenomenon is consistent with our daily bus ride experience, so it also verifies the validity of the model in Section 2.2.

Scenario 2 (Figure $4(\mathrm{~d})$ ) reveals that even if the speed of buses between two stations remains unchanged, the unreliability also occurs under the condition that the passenger arrival rate is unstable, and it spreads further backward. Such a conclusion is drawn out of Figure 4(d) that the headway of the bus in the first few stations changes rapidly because there are more bus passengers in the first few stations. Therefore, we believe that the operation of buses is affected by the 
TABLE 6: Scenarios description.

\begin{tabular}{lcc}
\hline Scenario & Speed & Passenger arrival rate \\
\hline Scenario 1 & Data from smart card data & Data from smart card data \\
Scenario 2 & $8 \mathrm{~m} / \mathrm{s}$ & Data from smart card data \\
Scenario 3 & Data from smart card data & Passenger arrival rate at any time is unchanged \\
\hline
\end{tabular}

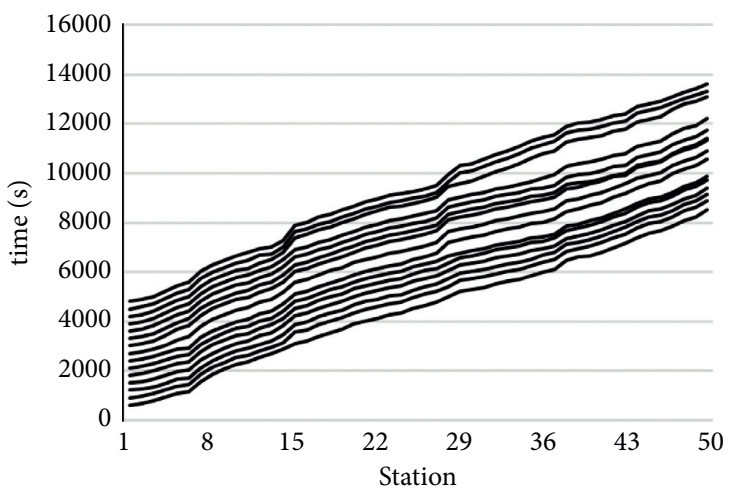

(a)

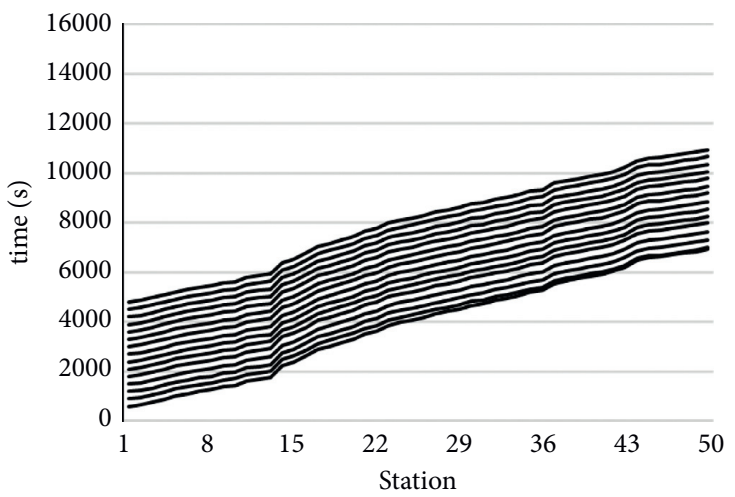

(c)

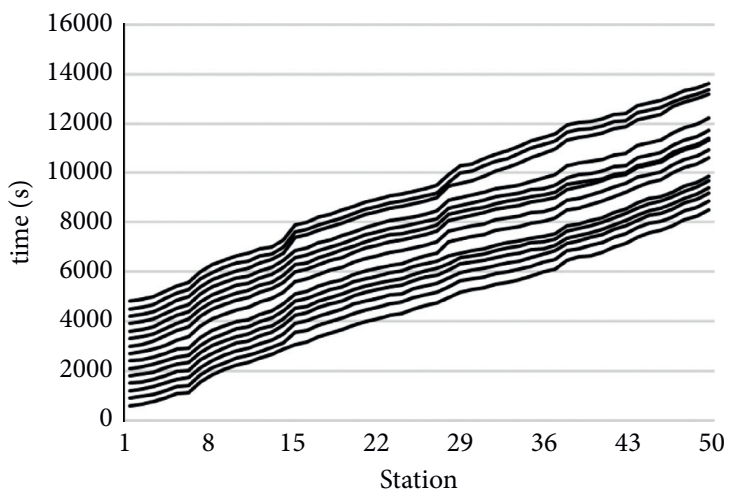

(e)

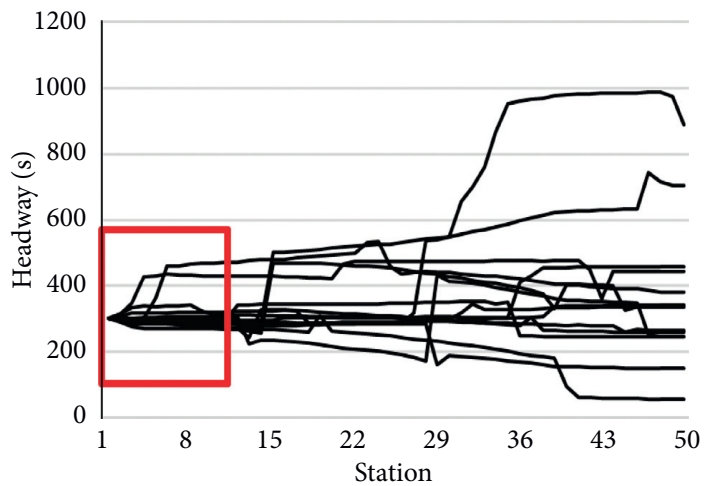

(b)

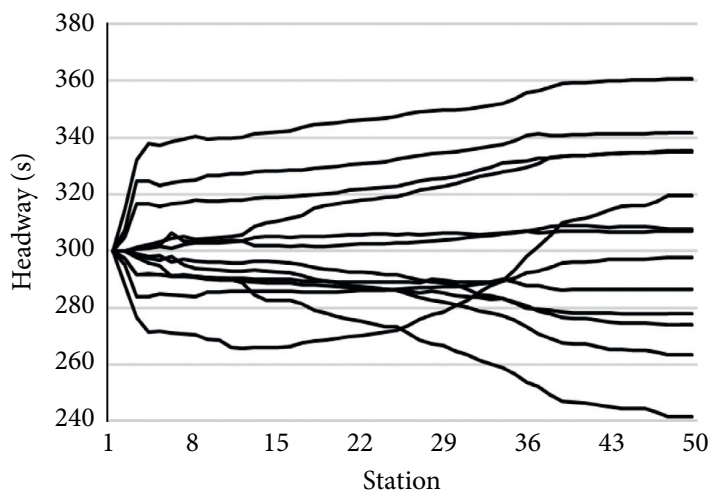

(d)

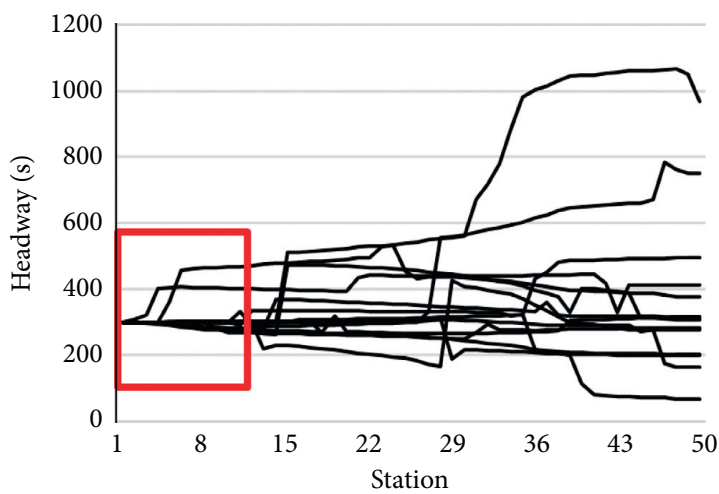

(f)

FIGURE 4: Simulation results in three scenarios: (a) bus trajectories for scenario 1 and (b) headway for scenario 1; (c) bus trajectories for scenario 2 and (d) headway for scenario 2; (e) bus trajectories for scenario 3 and (f) headway for scenario 3.

number passengers boarding and getting off in a certain degree. Unbalanced passenger distribution results in more unreliability.

By comparing scenario 3 (Figure 4(f)) with scenario 1 (Figure 4(b)), it is found that bus travel speed has a higher impact on reliability. When the passenger arrival rate does not change, the operation of the bus is still highly unreliability due to the change of bus travel speed. If the traffic status can be changed or the bus can adjust its traveling speed, the reliability of the bus will be changed. The method of bus speed control has been studied in previous studies $[26,27]$. The best case is that the bus speed can be controlled 
precisely like the subway and travel with a certain headway. That means, bus lanes may be helpful in improving reliability.

Unfortunately, it is difficult to change the speed of driving freely when buses travel in ordinary roads. In scenario 3, buses travel more reliable in the first few stops comparing with scenario 1 . This phenomenon can be found from red box in Figures 4(b) and 4(f). That means, if the passenger arrival rate does not change, it will help to improve reliability, and it has been proved in scenario 2 . It shows that the number of onboarding passengers has an impact on the operation of public transport. Moreover, when the number of passengers increases and the arrival rate changes more obviously, it will have a greater impact on the operational reliability just like the result shown in scenario 2 (Figure 4(d)).

In this paper, we focus on releasing the uneven distribution of passengers during peak periods to improve bus operation reliability. A short-turn strategy is proposed. But, different departure time and operation area of short-turn vehicles can make different influences on bus operational reliability. In the next section, we will discuss how to design a short-turn strategy to improve bus operation reliability.

\section{Short-Turn Strategy}

The most important factor to design a short-turn strategy is to establish the operating area and departure time of the short-turn vehicles. This study suggests that the operating area of short-turn vehicles should be the most concentrated section of the passenger's OD distribution, and the departure time of short-turn vehicles should be at the change point of speed or passenger arrival rate. These methods will be explained in detail in this section.

4.1. Operating Area. When we can get the OD data of the bus line based on smart card data, the interval of stations with larger passenger demand is selected out of OD data as the operating area of short-turn vehicles.

The operating area should satisfy two conditions simultaneously. Firstly, the ratio of OD in the selected area to the total OD of the line is larger, defined as $p_{i}^{1}$. Secondly, the ratio of $\mathrm{OD}$ in the selected area to the total number of passengers on board in the area is larger, defined as $p_{i}^{2}$ (in some cases, these two conditions may not be met simultaneously; selection should be done according to the actual situation because the passenger's demand in some lines is more evenly distributed). This method is described as follows:

Step1: given the parameter $u$, which is the number of stations that short-turn vehicles should operate.

Step2:

$i=0$

repeat

$i=i+1$

if $i<s-u$ then

$$
\begin{aligned}
& \qquad p_{i}^{1}=\left(\sum_{a=i}^{i+u} \sum_{b=i}^{i+u+1} \mathrm{OD}_{a b} / \sum_{a=i}^{s} \sum_{b=i}^{s} \mathrm{OD}_{a b}\right) \\
& \text { and } p_{i}^{2}=\left(\sum_{a=i}^{i+u} \sum_{b=i}^{i+u+1} \mathrm{OD}_{a b} / \sum_{a=i}^{s} \sum_{b=i}^{s} \mathrm{OD}_{a b}\right) \\
& \text { else } \\
& \quad \text { end if }
\end{aligned}
$$

Step3: we can get set 1 and set 2 from step2, where set $1=$ $\left(p_{i}^{1}, p_{i+1}^{1}, \ldots, p_{s-u}^{1}\right)$ and $\operatorname{set} 2=\left(p_{i}^{2}, p_{i+1}^{2}, \ldots, p_{s-u}^{2}\right)$.

Get the larger values in set 1 and set2. When the values can be found at station $i$, we can conclude that the operating area is $\mathrm{i}-i+u$.

4.2. Departure Time. The optimal frequency of the combination of the short-turn vehicles and the full-length vehicles has been discussed in previous studies. But, most of them did not give a concrete method to settle the departure time of short-turn buses. The operation of public transport is greatly impacted by the state of traffic flow and the demand of passengers; thus, this research believes that the departure time of the short-turn vehicle should be the time point at which the traffic flow speed is reduced and the passenger demand increases.

The cumulative sum control chart (CUSUM) method in change point theory can be applied to find the time, which is widely used in economics, meteorology, and other fields. The concrete calculation method is described as follows [20].

Assume there is a time series $X_{1}, X_{2}, X_{3}, \ldots, X_{T}$, and the change point can be found by 5 steps.

Step 1. We can calculate the average value of the series $\bar{X}=\operatorname{sum}\left(X_{1}, X_{2}, X_{3} \ldots X_{T}\right) / T$.

Step 2. Start the cumulative sum at zero by setting $S_{0}=0$.

Step 3. Calculate the cumulative sums of differences between the values and the average with formula $S_{i}=S_{i-1}+\left(X_{i}-\bar{X}\right)$. A series can be calculated. Then, we can get $S_{\text {diff }}=S_{\max }-S_{\min }$.

Step 4. Generate a bootstrap sample, which means to reorder the origin series randomly. Then, return step2, step 3, and a new difference of the bootstrap CUSUM can be calculated as $S_{\text {diff }}^{1}$.

Step 5. Repeat step 4 and get more $S_{\text {diff }}^{1}$, and the number of repetitions is $N$. Let $X$ be the number of bootstraps for which $S_{\text {diff }}^{1}<S_{\text {diff }}$. Then, the confidence level that a change occurred as a percentage is calculated as follows:

$$
\text { confidence level }=100 \frac{X}{N} \% \text {. }
$$

It is generally believed that if the confidence level is over $90 \%$, a change point can be detected in the series. A better estimate can be obtained by increasing the number of $N$. However, 1000 bootstraps are sufficient for most purposes. Once a change point is detected, $S_{m}=\max _{i=0,1, \ldots, T}\left|S_{i}\right|$ can be calculated. The point $m$ is estimated to be the last point before the change occurs. It can be considered as the departure time of short-turn vehicles. 


\section{Result}

5.1. Short-Turn Strategy. Passenger OD data of bus line No. 973 are selected to analyze, and the method has been proposed in Section 4.1 to determine the starting station and the terminal station of short-turn vehicles. Considering the passenger demand and the total length of the line, we take $u$ value of 12 as an example. The values of set 1 and set 2 can be calculated, as shown in Table 7.

In Table 7, the value of set 2 is also larger when set 1 is the largest at the first value. The value of set1 means that the number of passengers boarded on 1-12 station accounts for $22 \%$ of the total number of passengers on the entire line. The value of set2 means that passengers who boarded on station 1-12 and still get off on station 1-12 account for $56 \%$ of a total number of passengers on station 1-12. Therefore, the operation area of short-turn vehicles is set to station 1-12. Next, we need to determine the departure time of short-turn vehicles.

The analysis in previous scenarios shows that the bus operation is greatly affected by the traffic flow (speed). Therefore, this study takes the change point of the bus travel speed as a condition for judging the departure of short-turn vehicles. Taking the speed of the second interval as an example, the CUSUM method is applied to calculate accumulation difference of this series. The result is shown in Figure 5, and $S_{\text {diff }}=10.84$.

The original series is reordered to get $S_{\text {diff }}^{1}, S_{\text {diff }}^{2}, S_{\text {diff }}^{3}, \ldots, S_{\text {diff }}^{1000}$, and there are 925 of them less than

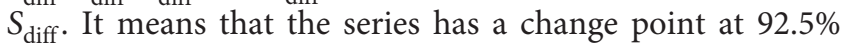
confidence level. When $S_{m}=\max _{i=0,1, \ldots, T}\left|S_{i}\right|$, the change point is $m$. There are 35 values in the series, which means there are 35 ! cases of random ordering. In this research, 1000 of them are selected at random. We can get different results for different bootstraps, and the random progress is repeated 10 times to prove that the confidence level can meet the requirements. The results are $91.8 \%, 92.3 \%, 89.9 \%, 92.1 \%$, 91.6, 92.5\%, 91.8\%, 90.6\%, 92\%, and 93.1\%.

There are 15 buses in the simulation, and the departure frequency is set to $300 \mathrm{~s}$. The departure time of the last bus should be at $4500 \mathrm{~s}$. Because speed changes every $600 \mathrm{~s}$, $4500 \mathrm{~s}$ corresponds to the 8 th time periods. In the first 8 periods, the cumulative sum $S_{5}=1.97$ is the maximum, which means the change point of speed at fifth time periods.

The cumulative sum series of the first 4 station intervals is calculated with the same method. As shown in Figure 6, change points are found in these 4 series, all of them at the same periods. We can confirm that the change points occur in 5th period. That is, the departure time of short-turn vehicles should be in the 5th period (2400-3000 s).

According to the hypothesis in Section 2, when a shortturn vehicle is in operation, passengers can choose either a short-turn vehicle or a full-length vehicle. It is generally believed that passengers choose the bus that arrives first. Passengers continue to accumulate during $H_{i}^{n-1, n}$. When there is one short-turn vehicle between bus $n-1$ and $n$, not all the accumulated passengers are suitable for the short-turn vehicle. In order to ensure the short-turn vehicle takes more passengers without causing waste, the departure time of the
Table 7: Passenger ratio data.

\begin{tabular}{|c|c|c|}
\hline Starting station & set1 & set2 \\
\hline 1 & 0.22 & 0.56 \\
\hline 2 & 0.19 & 0.55 \\
\hline 3 & 0.12 & 0.45 \\
\hline 4 & 0.11 & 0.45 \\
\hline 5 & 0.11 & 0.44 \\
\hline 6 & 0.1 & 0.43 \\
\hline 7 & 0.1 & 0.42 \\
\hline 8 & 0.09 & 0.4 \\
\hline 9 & 0.1 & 0.42 \\
\hline 10 & 0.09 & 0.38 \\
\hline 11 & 0.08 & 0.35 \\
\hline 12 & 0.09 & 0.35 \\
\hline 13 & 0.07 & 0.31 \\
\hline 14 & 0.06 & 0.26 \\
\hline 15 & 0.07 & 0.27 \\
\hline 16 & 0.08 & 0.29 \\
\hline 17 & 0.08 & 0.29 \\
\hline 18 & 0.08 & 0.33 \\
\hline 19 & 0.09 & 0.35 \\
\hline 20 & 0.1 & 0.37 \\
\hline 21 & 0.09 & 0.37 \\
\hline 22 & 0.1 & 0.37 \\
\hline 23 & 0.1 & 0.37 \\
\hline 24 & 0.09 & 0.34 \\
\hline 25 & 0.09 & 0.33 \\
\hline 26 & 0.08 & 0.31 \\
\hline 27 & 0.09 & 0.34 \\
\hline 28 & 0.09 & 0.36 \\
\hline 29 & 0.09 & 0.4 \\
\hline 30 & 0.11 & 0.47 \\
\hline 31 & 0.11 & 0.5 \\
\hline 32 & 0.11 & 0.55 \\
\hline 33 & 0.11 & 0.61 \\
\hline 34 & 0.1 & 0.66 \\
\hline 35 & 0.11 & 0.79 \\
\hline 36 & 0.1 & 0.88 \\
\hline 37 & 0.09 & 0.92 \\
\hline 38 & 0.08 & 0.98 \\
\hline 39 & 0.06 & 1 \\
\hline
\end{tabular}

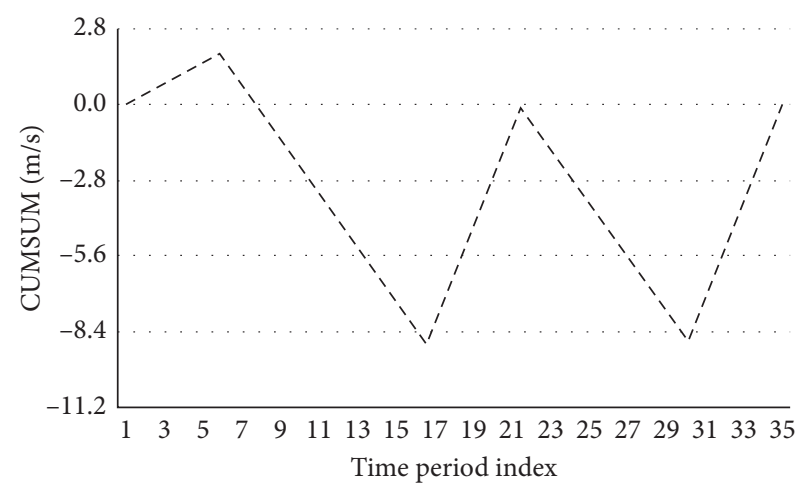

FIGURE 5: CUSUM chart of the second station interval.

short-turn vehicle should be as close as possible to bus $n$, rather than completely in the middle of bus $n-1$ and $n$ [7]. We assume that the two full-length vehicles will depart at $0 \mathrm{~s}$ 


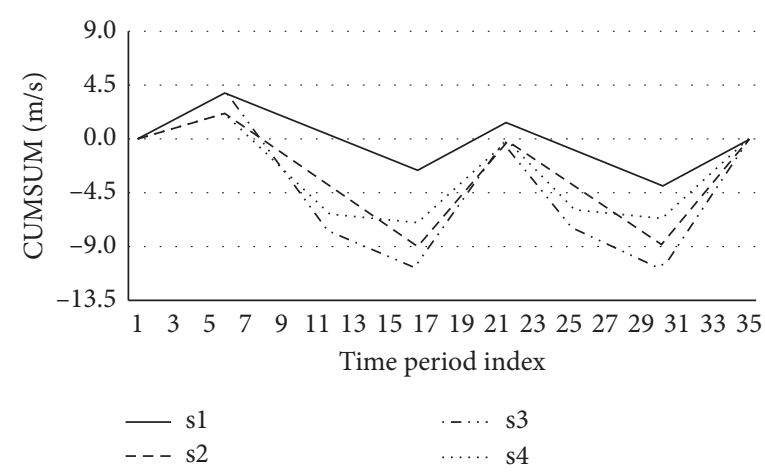

FIgURE 6: CUSUM charts of the first 4 station interval.

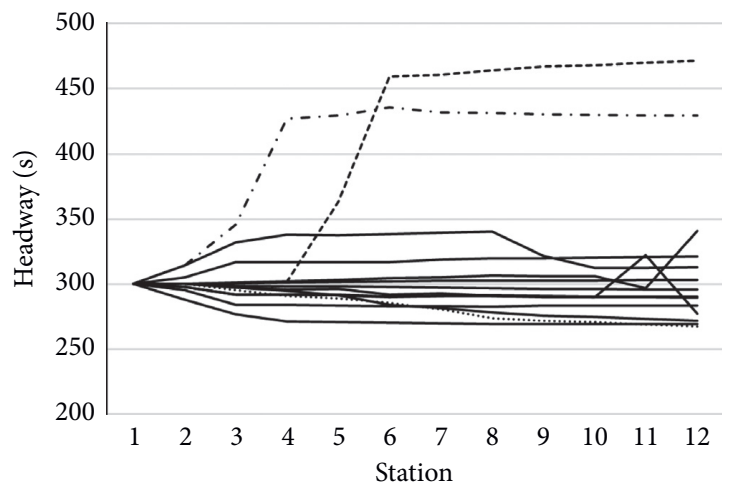

(a)

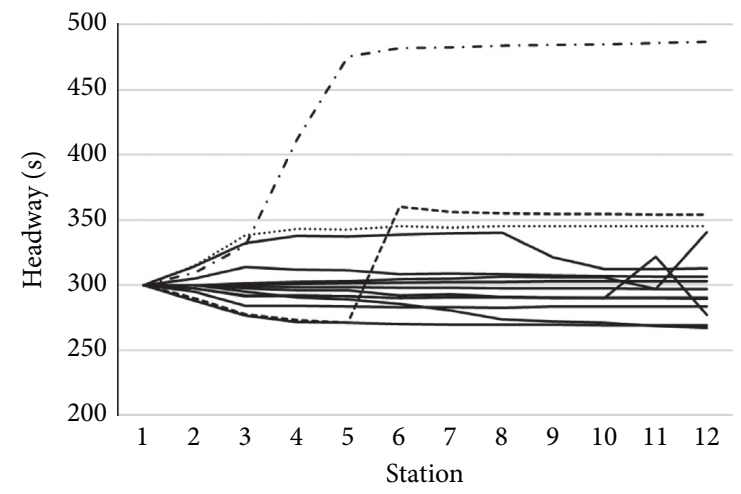

(b)

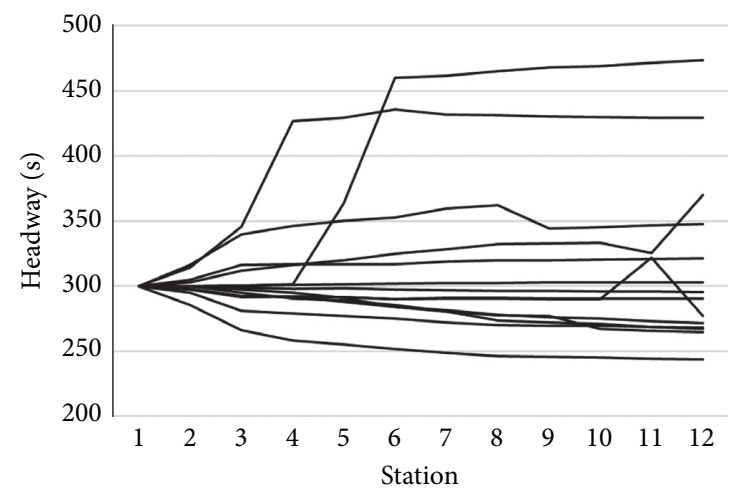

(c)

Figure 7: (a) Strategy A, (b) strategy B (dotted lines represent the headway of the full-length vehicles which adjoins the short-turn vehicles), and (c) strategy C.

and 300s, respectively. The departure time of the short-turn vehicle should be after $150 \mathrm{~s}$. Therefore, in this case study, the departure time of short-turn vehicles should be set at the $2600 \mathrm{~s}$ and $2900 \mathrm{~s}$ because the departure time of the adjacent full-length vehicles is $2400 \mathrm{~s}, 2700 \mathrm{~s}$, and $3000 \mathrm{~s}$. We define it as strategy B. The basic strategy is defined as strategy A (without short-turn vehicles). Meanwhile, in order to verify the rationality of dispatch the short-turn vehicles at the change point, another departure plan is given randomly, for example, the departure time of short-turn vehicles at the
$1100 \mathrm{~s}$ and $1700 \mathrm{~s}$. In addition, the departure time of the adjacent full-length vehicles is at $900 \mathrm{~s}, 1200 \mathrm{~s}, 1500 \mathrm{~s}$, and $1800 \mathrm{~s}$. The operation area is still in station $1-12$. We define it as strategy $\mathrm{C}$.

5.2. Simulation Result. These three strategies are simulated separately. The bus headway of station $1-12$ is shown in Figure 7 . The mean absolute error between bus headway and the departure interval is used to evaluate different strategies, which can be formulated as follows: 


$$
\text { MAE }=\frac{\sum_{n=2}^{15} \sum_{i=1}^{12}\left|H_{i}^{n-1, n}-H_{1}^{n-1, n}\right|}{15^{*} 12} .
$$

Through the numerical simulation results, it can be found that MAE of the first 12 stations is $46.9 \mathrm{~s}$ without the short-turn strategy being applied (strategy A), while the value changes to $44.7 \mathrm{~s}$ after the strategy $\mathrm{B}$ is employed. The overall reliability of the 15 buses in the system increases by $4.7 \%$. It can be seen from the result that strategy B can change full-length vehicles' operating state obviously, especially for these vehicles whose departure time are close to short-turn vehicles'. Dotted lines are used in Figure 7(b) to mark the three buses. The overall reliability of the first 12 stations of these three buses increased by $10.4 \%$. Meanwhile, the reliability of vehicles that depart further behind the short-turn vehicles will also be affected, but the effect will gradually weaken. For strategy C (Figure 7(c)), MAE is $50.2 \mathrm{~s}$, that means the headway distribution is more discrete than the other two strategies. The reliability is even lower than the without short-turn strategy case. Compared with the simulation results of the three strategies, it can be explained that if the short-turn strategy cannot be designed scientifically, it will lead to the reduction of the overall reliability of the bus operation. Therefore, the reasonable design of the departure plan for short-turn vehicles should be considered carefully by the bus operating company. One of the better options is that the short-turn vehicles depart at the change point in the peak period (Figure 7(b)) and operate in the area with larger passenger demand.

\section{Discussion and Conclusion}

6.1. Discussion. It can be seen from the result that we calculated the operating area should be from station 1 to 12 based on OD data (Section 5.1). However, there are some subjective factors to calculate this area using the method in Section 4.1 such as the value of $u$. In this paper, $u$ is set 12 because possible carrying capacity of the short-turn vehicle, total length of bus line, and turnover efficiency are considered. Different lines or cities may have different values.

Meanwhile, it should be noted that an implicit assumption in the simulation of this study is that once the change point is found, the short-turn vehicles can arrive at the target station. But, it seems to be unrealistic. Because the short-turn vehicles take a certain amount of time from the departure station to the target station, the travel time needs to be considered to achieve the desired improvement. If we can predict the traffic congestion state and the passenger flow more accurately through the forecasting method, we may be able to find a more suitable timetable of short-turn vehicles. Therefore, when the bus company uses this method, it is necessary to forecast the traffic state for a better shortturn strategy $[28,29]$.

The essence of the short-turn strategy is to adjust the number of passengers boarding full-length vehicles at some stations. That means, if the passenger number of each station can be well optimized, it will be helpful to improve the reliability of the bus operation. This view also provides a new idea for the operation and management of public transportation. If authorities hope to attract more passengers to choose to take the bus by improving bus operation reliability, not only the method of speed control and the express bus could be useful but the management of passenger demand is also very important. For example, we can restrict passenger flow at some stations to improve the stability of passenger numbers during peak hours [30, 31]. Also, we can use the transportation information system to publish real-time public transportation information such as the degree of congestion in the bus or bus arrival time to guide passenger demand [32].

The result shows in Section 3 that passenger demands may impact bus operation reliability. In addition, the larger the number of passengers, the greater the impact. This also implies that if this method is used in another bus line, it will have different effects. Therefore, we suggest that it would be better to adopt the short-turn strategy in the rush hours and the bus lines with more passengers. The result provides an idea to explain the capacity paradox of the transit system that has been proved [33], which may be caused by the timing of departure.

6.2. Conclusion. In this study, a bus operation state model considering the operation of short-turn vehicles was firstly established. Then, two parameters affecting the operation state of buses are analyzed, which are passenger arrival rate and bus travel speed. Result in Section 3 shows that passenger distribution affects the operating state of full-length vehicles in the system. Then, the short-turn strategy is proposed which contains two parts based on the number of passengers to determine the operation area of short-turn vehicles and the departure time using change point theory. Finally, the smart card data of buses are used to verify the proposed method through numerical simulation. The simulation result shows that if a short-turn strategy is designed by using the method mentioned in Section 4, the reliability of the full-length vehicles in the operation area of the shortturn vehicles increases by $4.7 \%$. But, if the departure time of short-turn vehicles is set randomly, it will reduce the reliability by $7 \%$, even the operating area is reasonable. Affected by passengers' boarding and dropping, the short-turn strategy may affect the operation of full-length vehicles in different degree. When the number of passengers increases and the arrival rate changes more obviously, the departure plan of short-turn vehicles will have a greater impact on the operation reliability of full-length vehicles.

By comparing with different departure strategies, we find that the departure time and operating area of short-turn vehicles determined in Section 4 are reasonable. It can optimize reliability to a certain degree. Meanwhile, the strategy can be deployed in real-time systems, and we can formulate the optimized short-turn strategy simply and quickly.

Since the short-turn vehicles generally run in the upward and downward directions, the short-turn strategy should have an impact on operating of full-length vehicles in two directions. But, this research only covers a certain direction of operation. In future research, the impact of the short-turn 
strategy on the entire public transport system in two directions shall be touched. In addition, this study only considers short-turn vehicles depart at the change point. The optimization of the cost is not considered, such as passengers' waiting time and bus company management cost. Also, sensitivity analysis of bus frequency and passengers demand need to be considered. These points will also be supplemented in future research.

\section{Data Availability}

The IC card data used to support the findings of this article are available from the corresponding author upon request.

\section{Conflicts of Interest}

The authors declare that they have no conflicts of interest.

\section{Acknowledgments}

This research was funded by the National Natural Science Foundation of China (nos. U1811463, 51878020, 51908018, and 51308018) and Natural Science Foundation of Hebei Province (no. E2018407051). Useful suggestions were given by Dr. Yue Liu from the University of Wisconsin-Milwaukee and Dr. Huanmei Qin from the Beijing University of Technology which are also acknowledged.

\section{References}

[1] J. A. Sorratini, R. Liu, and S. Sinha, "Assessing bus transport reliability using micro-simulation," Transportation Planning and Technology, vol. 31, pp. 303-324, 2008.

[2] J. Bates, J. Polak, P. Jones, and A. Cook, "The valuation of reliability for personal travel," Transportation Research Part E: Logistics and Transportation Review, vol. 37, no. 2-3, pp. 191-229, 2001.

[3] M. A. Turnquist and L. A. Bowman, "The effects of network structure on reliability of transit service," Transportation Research Part B: Methodological, vol. 14, no. 1-2, pp. 79-86, 1980.

[4] X. Chen, L. Yu, Y. Zhang, and J. Guo, "Analyzing urban bus service reliability at the stop, route, and network levels," Transportation Research Part A: Policy and Practice, vol. 43, no. 8, pp. 722-734, 2009.

[5] A. Mark, E. Amir, and E. Israel, "Optimal control of headway variation on transit routes," Journal of Advanced Transportation, vol. 20 , pp. 73-88, 2010.

[6] P. R. Tétreault and A. M. El-Geneidy, "Estimating bus run times for new limited-stop service using archived AVL and APC data," Transportation Research Part A: Policy and Practice, vol. 44, no. 6, pp. 390-402, 2010.

[7] P. G. Furth, "Zonal route design for transit corridors," Transportation Science, vol. 20, no. 1, pp. 1-12, 1986.

[8] S. Liang, S. Zhao, C. Lu, and M. Ma, "A self-adaptive method to equalize headways: numerical analysis and comparison," Transportation Research Part B: Methodological, vol. 87, pp. 33-43, 2016.

[9] S. Liang, M. Ma, and S. He, "Multiobjective optimal formulations for bus fleet size of public transit under headwaybased holding control," Journal of Advanced Transportation, vol. 2019, Article ID 2452348, 14 pages, 2019.
[10] S. Liang, M. Ma, S. He, H. Zhang, and P. Yuan, "Coordinated control method to self-equalize bus headways: an analytical method," Transportmetrica B: Transport Dynamics, vol. 7, no. 1, pp. 1175-1202, 2019.

[11] A. Ceder, "Optimal design of transit short-turn trips," Transportation Research Record Journal of the Transportation Research Board, vol. 1221, pp. 8-22, 1989.

[12] P. D. Site and F. Filippi, "Service optimization for bus corridors with short-turn strategies and variable vehicle size," Transportation Research Part A: Policy and Practice, vol. 32, pp. 19-38, 1998.

[13] C. E. Cortés, S. Jara-Díaz, and A. Tirachini, "Integrating short turning and deadheading in the optimization of transit services," Transportation Research Part A: Policy and Practice, vol. 45, no. 5, pp. 419-434, 2011.

[14] A. Tirachini, C. E. Cortés, and S. R. Jara-Díaz, "Optimal design and benefits of a short turning strategy for a bus corridor," Transportation, vol. 38, no. 1, pp. 169-189, 2011.

[15] P. G. Furth, "Short turning on transit routes," Transportation Research Record Journal of the Transportation Research Board, vol. 1108, pp. 42-52, 1987.

[16] D. Canca, E. Barrena, G. Laporte, and F. A. Ortega, "A shortturning policy for the management of demand disruptions in rapid transit systems," Annals of Operations Research, vol. 246, no. 1-2, pp. 145-166, 2016.

[17] A. Ceder, S. Hassold, and B. Dano, "Approaching even-load and even-headway transit timetables using different bus sizes," Public Transport, vol. 5, pp. 193-217, 2016.

[18] H. Zhang, S. Zhao, H. Liu, and L. Jin, "A dynamic shortturning bus control for uncertain demand," Journal of Advanced Transportation, vol. 2017, Article ID 7392962, 9 pages, 2017.

[19] G. F. Newell, "Dispatching policies for a transportation route," Transportation Science, vol. 5, no. 1, pp. 91-105, 1971.

[20] S. J. Berrebi, E. Hans, N. Chiabaut, J. A. Laval, L. Leclercq, and K. E. Watkins, "Comparing bus holding methods with and without real-time predictions," Transportation Research Part C: Emerging Technologies, vol. 87, pp. 197-211, 2018.

[21] W. A. Taylor, "Change-point analysis: a powerful new tool for detecting changes," 2000, http://www.variation.com/files/ articles/changepoint.pdf.

[22] L. Horváth and M. Hušková, "Change-point detection in panel data," Journal of Time Series Analysis, vol. 33, no. 4, pp. 631-648, 2012.

[23] B. Safari, "Trend analysis of the mean annual temperature in Rwanda during the last fifty two years," Journal of Environmental Protection, vol. 3, no. 6, pp. 538-551, 2012.

[24] Y. Ji, X. Yang, and Y. Du, "Optimal design of a short-turning strategy considering seat availability," Journal of Advanced Transportation, vol. 50, no. 7, pp. 1554-1571, 2016.

[25] Y. Zhou, L. Yao, Y. Chen et al., "Bus arrival time calculation model based on smart card data," Transportation Research Part C: Emerging Technologies, vol. 74, pp. 1634-1638, 2016.

[26] C. F. Daganzo and J. Pilachowski, "Reducing bunching with bus-to-bus cooperation," Transportation Research Part B: Methodological, vol. 45, no. 1, pp. 267-277, 2011.

[27] W. Wu, W. Ma, K. Long et al., "Designing sustainable public transportation: integrated optimization of bus speed and holding time in a connected vehicle environment," Sustainability, vol. 8, 1170 pages, 2016.

[28] Y. Bai, Z. Sun, B. Zeng et al., "A multi-pattern deep fusion model for short-term bus passenger flow forecasting," Applied Soft Computing, vol. 58, pp. 669-680, 2016. 
[29] M. M. Rahman, S. C. Wirasinghe, and L. Kattan, "Analysis of bus travel time distributions for varying horizons and realtime applications," Transportation Research Part C: Emerging Technologies, vol. 86, pp. 453-466, 2018.

[30] C. F. Daganzo, "A headway-based approach to eliminate bus bunching: systematic analysis and comparisons," Transportation Research Part B: Methodological, vol. 43, no. 10, pp. 913-921, 2009.

[31] H. Zhang, S. Zhao, H. Liu et al., "Design of limited-stop service based on the degree of unbalance of passenger demand," PLoS One, vol. 13, no. 3, 2018.

[32] G. Gentile, S. Nguyen, and S. Pallottino, "Route choice on transit networks with online information at stops," Transportation Science, vol. 39, no. 3, pp. 289-297, 2005.

[33] Y. Jiang and W. Y. Szeto, "Reliability-based stochastic transit assignment: formulations and capacity paradox," Transportation Research Part B: Methodological, vol. 93, pp. 181206, 2016. 